\title{
Hybrid light-matter networks of Majorana zero modes
}

\author{
L. C. Contamin ${ }^{1}$, M. R. Delbecq ${ }^{1}$, B. Douçot ${ }^{2}$, A. Cottet $\mathbb{B}^{1 凶}$ and T. Kontos ${ }^{1 凶}$
}

Topological excitations, such as Majorana zero modes, are a promising route for encoding quantum information. Topologically protected gates of Majorana qubits, based on their braiding, will require some form of network. Here, we propose to build such a network by entangling Majorana matter with light in a microwave cavity QED set-up. Our scheme exploits a light-induced interaction which is universal to all the Majorana nanoscale circuit platforms. This effect stems from a parametric drive of the lightmatter coupling in a one-dimensional chain of physical Majorana modes. Our set-up enables all the basic operations needed in a Majorana quantum computing platform such as fusing, braiding, the crucial T-gate, the read-out, and importantly, the stabilization or correction of the physical Majorana modes.

npj Quantum Information (2021)7:171 ; https://doi.org/10.1038/s41534-021-00508-w

\section{INTRODUCTION}

Majorana quasiparticles in condensed matter systems have been the subject of intense experimental work for almost a decade ${ }^{1-5}$, for their potential in defining topologically protected qubits and gates $^{6}$. However, experimental realizations have not succeeded so far in measuring the expected non-abelian statistics of these exotic excitations. Several protocols have been proposed for performing such advanced experiments through electronic transport measurements ${ }^{6-14}$. They all require a microscopic control and fine tuning of the experimental platforms, a two-dimensional (2D) or at least a network geometry and an invasive transport-based read-out.

Cavity photons have appeared as a major tool for manipulating, coupling and reading out the quantum state of superconducting circuits $^{15-17}$. However, the direct application of circuit quantum electrodynamics (CQED) techniques to Majorana bound states (MBSs) is hindered by parity conservation, which forbids a direct energy exchange between an isolated Majorana doublet and a cavity $^{18}$. It was proposed to probe the presence and parity of a given Majorana doublet by observing transitions to supplementary states $^{18-20}$ or by using a charge-sensitive Josephson circuit ${ }^{21}$. In principle, one can also detect the dynamical phase resulting from the braiding of Majorana modes by probing the cavity field ${ }^{22}$. In that particular proposal, braiding is assumed to pre-exist and the cavity signal is used solely as a probe. No specific solution is provided for universal quantum computation. Overall, the above proposals do not provide a way to manipulate and couple Majorana states through the photonic degree of freedom. This is why CQED has not been envisioned as a full platform for performing all the requested operations for fusing and braiding the MBSs, so far.

In this paper, we propose a hybrid Majorana-cavity platform that fills these gaps. We show that by modulating the Majorana-photon coupling at the cavity frequency, one can fuse and braid two MBSs or perform T-gates in a four to six MBS linear chain, enabling universal quantum computation. This resource is obtained because the modulation produces an effective reconfigurable array of one-dimensional (1D) chains connected by triangular nodes out of a 1D chain. Finally, we show how we can preserve the topological protection of the Majorana modes using an active stabilization based on the joint action of the cavity photons and the modulation of the coupling.

\section{RESULTS}

We consider a linear chain of MBSs hosted in a nanoconductor embedded in a photonic cavity, as represented in Fig. 1a. The elementary operations used throughout the paper and their effect on the cavity field are depicted in Fig. 1b-e. A possible example of network obtained with our scheme is depicted in Fig. 1f. We consider a single photonic mode $\hat{a}$ with frequency $\omega_{\mathrm{c}} / 2 \pi$. Each MBS is associated with a self-adjoint creation operator $\hat{\gamma}_{i}$ as depicted in Fig. 1a. A small overlap between two neighboring MBSs, defining the section $j j+1$, gives rise to energy splittings $\epsilon_{j j+1}$, which are exponentially suppressed with the distance between the two MBSs. The low-energy effective Hamiltonian of the system can be written $\mathrm{as}^{23}$ :

$$
\bar{H}_{\text {chain }}=\sum_{j} g_{j j+1}\left(\hat{a}+\hat{a}^{\dagger}\right) i \hat{\gamma}_{j} \hat{\gamma}_{j+1}+\sum_{j} \epsilon_{j j+1} i \hat{\gamma}_{j} \hat{\gamma}_{j+1}+\hbar \omega_{c} \hat{a}^{\dagger} \hat{a}
$$

One can associate to each Majorana pair $(j, k)$ a topological charge with a parity operator $\hat{P}_{j k}=i \hat{\gamma}_{j} \hat{\gamma}_{k}$. Unless otherwise specified, we assume that our chain has Majorana modes with energies $\epsilon_{j j+1}$ much smaller than $\hbar \omega_{c}$.

One of the main results of our work is how we can shape the above Hamiltonian to manipulate, read-out and stabilize Majorana modes under a parametric drive. The electron-photon couplings can be locally modulated at microwave frequencies $\omega_{\mathrm{RF}} \simeq \omega_{\mathrm{c}}$ through a modulation of local gate electrodes (which modulate the MBS overlap) such that: $g_{j i+1}(t)=\bar{g}_{j i+1}+\tilde{g}_{j j+1} \cos \left(\omega_{\mathrm{RF}} t+\phi_{i j+1}\right)$. In this case, one has to slightly turn off the topological protection (which can be restored with the scheme explained at the end of the paper). This means that one has typically $g_{i j+1} \approx \epsilon_{i j+1}$. Having in mind a low dimensional material in proximity with a superconductor, the physical electrical dipole allowing such a modulation can be induced by attaching galvanically the superconductor to the central conductor of the cavity. This corresponds to the dipole formed at the barrier between the superconductor and the low dimensional system. The modulation can be achieved here by modulating the electric potential of gate electrodes. In the following, we consider different types of parametric drives to implement the different Majorana operations. In all cases, we can transform Hamiltonian (1) into a quasistatic one by going into the rotating frame of the cavity field and/or performing a suitable dispersive unitary transformation. In order to be more specific, we first discuss the case of two or four MBSs.

'Laboratoire de Physique de l'Ecole Normale Supérieure, ENS, Université PSL, CNRS, Sorbonne Université, Université Paris-Diderot, Sorbonne Paris Cité, Paris, France. ${ }^{2}$ Laboratoire de Physique Théorique et des Hautes Energies, CNRS, Sorbonne Paris Cité, Paris, France. ${ }^{凶}$ email: audrey.cottet@ens.fr; takis.kontos@ens.fr 

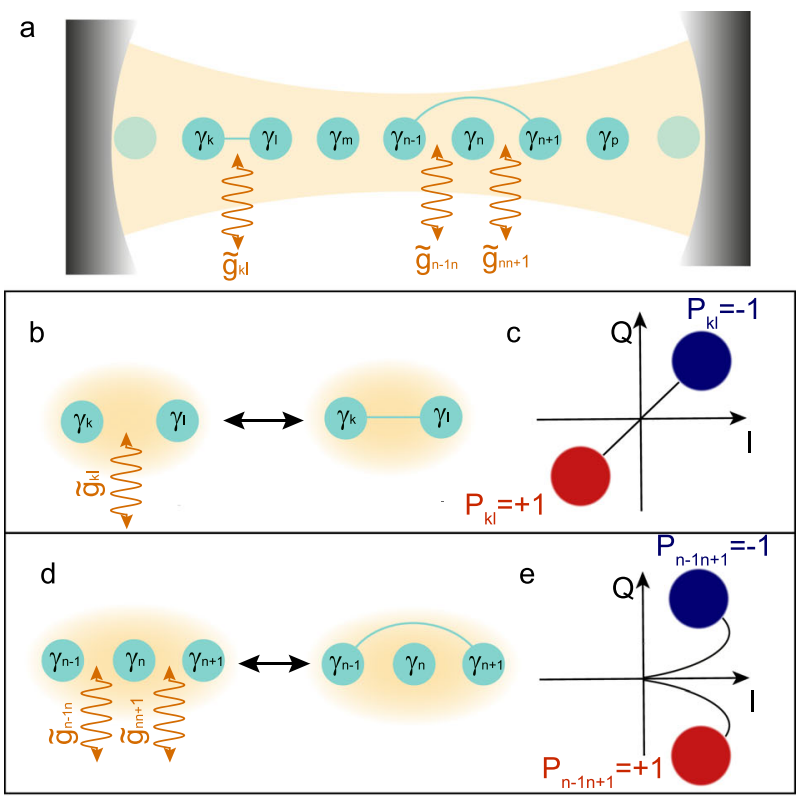

f

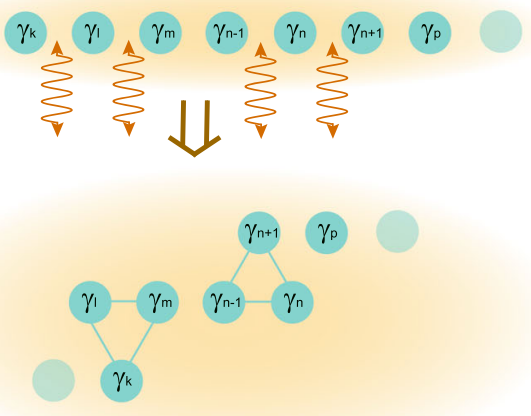

Fig. 1 Hybrid light-matter network of Majorana zero modes. a We consider a chain of Majorana quasiparticles $\hat{\gamma}_{j}$ embedded inside a microwave cavity. The cavity is represented as the two mirrors (shaded black) concentrating a photonic field (light yellow) around the circuit. The Majorana modes are represented as turquoise balls. The microwave drive of each section $(j, j+1)$ is represented as orange vertical wavy lines. The resulting effective interaction is represented as turquoise lines, turning the chain into an elementary network suitable for fusing, braiding, and stabilizing Majorana modes. b Nearest neighbor interaction and its signature in the trajectory of a coherent state in the quadrature $I-Q$ plane of the cavity field (c). d Second nearest neighbor interaction and its signature in the trajectory of a coherent state in the quadrature $I-Q$ plane of the cavity field (e). $\mathbf{f}$ Example of transformation of the 1D chain into a reconfigurable array of 1D chains used throughout the paper using our parametric excitation scheme.

\section{Nearest neighbor light bonds}

We specialize here the discussion to the section delimited by Majorana modes 1 and 2. The low-energy Hamiltonian of the system can be reduced to:

$H_{\text {chain }}=i \epsilon_{12}(t) \hat{\gamma}_{1} \hat{\gamma}_{2}+i g_{12}(t) \hat{\gamma}_{1} \hat{\gamma}_{2}\left(\hat{a}+\hat{a}^{\dagger}\right)+\hbar \omega_{\mathrm{c}} \hat{a}^{\dagger} \hat{a}$

We can rewrite the Hamiltonian in a rotating frame at $\omega_{c}$. The corresponding unitary transformation reads : $U_{j j+1}=e^{i \omega_{\mathrm{RF}} \hat{a}^{\dagger} \hat{a} t}$ with $j=1$. We obtain:

$\tilde{H_{\mathrm{eff}}} \approx \frac{1}{2} \tilde{g}_{12}\left(e^{i \phi_{12}} \hat{a}^{\dagger}+e^{-i \phi_{12}} \hat{a}\right) i \hat{\gamma}_{1} \hat{\gamma}_{2}$ where the static term proportional to $\bar{g}_{12}$ is neglected as a fast oscillating term, under the rotating wave approximation (RWA). We define the two quadratures of the cavity field $: \hat{l}=\hat{a}+\hat{a}^{\dagger}$ and $\hat{Q}=i\left(\hat{a}-\hat{a}^{\dagger}\right)$, the expectation values of which allow us to define the $I-Q$ plane. These components of the electromagnetic field correspond to the in-phase and out-of-phase microwave signal of the cavity and can be measured by homodyne or heterodyne detection ${ }^{15}$. Equation (3) shows an effective coupling between the Majorana's 1 and 2 which can be used to measure their parity $\hat{P}_{12}=i \hat{\gamma}_{1} \hat{\gamma}_{2}$ via the cavity field as shown in Fig. 1. This measurement follows the same principle as the longitudinal coupling read-out for qubits ${ }^{24}$. The parity eigenstate is read-out from the position of the coherent state spots in the $I-Q$ plane associated with the $\hat{P}_{12}= \pm 1$ eigenvalues. The contrast for the coherent state spots in the $I-Q$ plane is $\tilde{g}_{12} / \sqrt{\kappa}$, where $K$ is the damping rate of the cavity (see "Input-output theory" section of "Methods"), which can be made much larger than the width of the Gaussian spots of the coherent states even deep in the topological regime where $\tilde{g}_{12} \rightarrow 0$ for small enough $\kappa$. Similarly, one can also measure $\hat{P}_{23}=i \hat{\gamma}_{2} \hat{\gamma}_{3}$. This is simply done by letting $\tilde{g}_{23}$ non zero keeping the other modulating terms negligible.

\section{Second nearest neighbor light bonds}

We now show specifically on a 4 Majorana chain $(1,2,3,4)$ how we can obtain a second nearest neighbor photon-mediated interaction between MBSs 2 and 4. Starting again from Hamiltonian (1), we now assume that the radio frequency (RF) signal acting on the gates $(2,3)$ and $(3,4)$ is detuned from the cavity. In order to understand the effect of this drive, one can perform the combined unitary transformation:

$U_{j, j+2}=e^{i \omega_{\mathrm{RF}} \hat{a}^{\dagger} \hat{a} t} e^{\left[\frac{\tilde{g}_{23}}{\hbar\left(\omega_{\mathrm{RF}}-\omega_{C}\right)}\left(e^{i \phi_{23}} \hat{a}^{\dagger}+e^{-i \phi_{23}} \hat{a}\right) i \hat{\gamma}_{2} \hat{\gamma}_{3}+\frac{\tilde{g}_{34}}{\hbar\left(\omega_{\mathrm{RF}}-\omega_{C}\right)}\left(e^{i \phi_{34}} \hat{a}^{\dagger}+e^{-i \phi_{34}} \hat{a}\right) \hat{\gamma}_{3} \hat{\gamma}_{4}\right]}$

with $j=2$. It combines the RWA in the frame of the gate drives and a dispersive transformation in $\tilde{g}_{23}, \tilde{g}_{34}$ which implies that $\omega_{\mathrm{RF}}-\omega_{\mathrm{c}} \gg \tilde{g}_{23}, \tilde{g}_{34}$. The outcome of these two transformations is:

$$
H=\hbar\left(\omega_{\mathrm{c}}-\omega_{\mathrm{RF}}\right) \hat{a}^{\dagger} \hat{a}+8 i \frac{\tilde{g}_{23} \tilde{g}_{34}}{\hbar\left(\omega_{\mathrm{RF}}-\omega_{\mathrm{c}}\right)} \sin \left(\phi_{23}-\phi_{34}\right) \hat{\gamma}_{2} \hat{\gamma}_{4}\left(\hat{a}^{\dagger} \hat{a}+1 / 2\right)
$$

The reason why $\hat{\gamma}_{3}$ does not appear in the effective Hamiltonian (5) can be intuitively explained as follows: each of the gates is acting on different directions of the effective Bloch sphere. The gate 23 is equivalent to a $\hat{\sigma}_{z}$ rotation whereas the gate 34 is equivalent to a $\sigma_{x}$ rotation. Since the two gates are driven with a phase shift $\phi_{23}-\phi_{34}$, the protocol is equivalent to a composition of $\hat{\sigma}_{z}$ and $\hat{\sigma}_{x}$, hence a $\hat{\sigma}_{y}$ rotation which is $\hat{\gamma}_{2} \hat{\gamma}_{4}$. This interaction for $\hat{\gamma}_{2} \hat{\gamma}_{4}$ is crucial for the braiding operation as we will see below.

\section{Quadrature selective couplings}

Another important interaction mediated by the cavity enables the implementation of the challenging T-gate, which corresponds to a $\pi / 8$ geometrical phase during the unitary evolution of the system. We specialize to the 4 MBS chain again for the sake of simplicity and assume that $\bar{\epsilon}_{34} \neq 0$ (hence we lift the topological protection here) and $\tilde{g}_{23} \neq 0$. With the unitary transformation $U_{T, j, j+1, j+2}=$ $e^{i\left(\omega_{c} \hat{a}^{\dagger} \hat{a}+\bar{\epsilon}_{34} i_{\hat{Y}_{3}} \hat{Y}_{4} t\right)}$ (interacting picture with $j=2$ ), the effective Hamiltonian becomes:

$$
\begin{aligned}
H_{\pi / 8}= & \frac{\tilde{g}_{23}}{2}\left(e^{i \omega_{c} t} \hat{a}^{\dagger}+e^{-i \omega_{c} t} \hat{a}\right) \cos \left(\omega_{\mathrm{RF}} t+\phi_{23}\right) \\
& \left\{\cos \left(2 \bar{\epsilon}_{34} t\right) i \hat{\gamma}_{2} \hat{\gamma}_{3}+\sin \left(2 \bar{\epsilon}_{34} t\right) i \hat{\gamma}_{3} \hat{\gamma}_{4}\right\}
\end{aligned}
$$


For $\omega_{\mathrm{RF}}=\omega_{\mathrm{c}}+2 \bar{\epsilon}_{34}$, retaining only the resonant terms, we get:

$H_{\pi / 8}=\frac{\tilde{g}_{23}}{2}\left(e^{i \phi_{23}} \hat{a}^{\dagger}+e^{-i \phi_{23}} \hat{a}\right) i \hat{\gamma}_{2} \hat{\gamma}_{3}+\frac{\tilde{g}_{23}}{2} i\left(e^{i \phi_{23}} \hat{a}^{\dagger}-e^{-i \phi_{23}} \hat{a}\right) i \hat{\gamma}_{2} \hat{\gamma}_{4}$

Such a form shows that the two different directions corresponding to $i \gamma_{2} \gamma_{3}$ or " $\sigma_{x}$ " and to $i \gamma_{2} \gamma_{4}$ or " $\sigma_{y}$ " become coupled with the two quadratures of the cavity field (respectively, $I$ and $Q$ ). This allows us to perform a T-gate simply by measuring the cavity field along the bisector between I and $Q$.

\section{General form of the chain-cavity Hamiltonian}

Combining the unitary transformations $U_{T, j, 1, j+2}, U_{j, j+2}$ and $U_{j, j+1}$ allows one to obtain a 2D network Hamiltonian of the form:

$H_{\text {eff }}=\sum_{n, m} i \hat{\gamma}_{n} \hat{\gamma}_{m} f_{n m}\left(\hat{a}^{\dagger}, \hat{a}, \hat{a}^{\dagger} \hat{a}\right)+\hbar \delta \hat{a}^{\dagger} \hat{a}$

where $f_{n m}$ is a linear combination of $\hat{a}, \hat{a}^{\dagger}, \hat{a}^{\dagger} \hat{a}$, which depends on the operation considered, and $\delta=\omega_{\mathrm{c}}-\omega_{\mathrm{RF}}$ is the detuning between the drive and the cavity. Simple examples are given in the previous sections in the specialized case of 2 to 4 Majorana modes. The different unitary transformations can be straightforwardly combined if the Majorana operators corresponding to the different sections of the chain are not present in two unitary operations. A particularly simple situation is that of dimerized chain in which every other section is "switched on," which corresponds to the situation depicted at the end of the main text. Because not only nearest neighbor interactions can be engineered but also the chain becomes an effective 2D network or, more accurately, a reconfigurable array of $1 \mathrm{D}$ chains connected by triangular nodes. The change from $1 D$ to $2 D$ is one of the main resources that we exploit in this paper. As shown in Fig. 1b-e, the read-out of the parity $\hat{P}_{i j+1}$, or $\hat{P}_{i j+2}$ of pairs $(j, j+1)$ or $(j, j+2)$ can be implemented by choosing appropriate gate voltage pulses (see "Methods").
The specific trajectories of the coherent cavity field carrying the information on the MBSs parity in the two elementary cases is shown in Fig. 1c, e. This information can be retrieved by measuring the field leaking out of the cavity with microwave techniques, as shown in the input-output theory section in the methods. Unless otherwise specified, we now assume that the chain considered has a given total even parity. In addition, we will omit for clarity the $j$ index of each Majorana until the discussion of the Majorana stabilization, replacing $j+1 . . j+6$ by $1 . .6$.

\section{Fusion protocol}

The fusion of two MBSs $\mathrm{j}$ and $\mathrm{k}$ requires the projective measurement of their parity $\hat{P}_{j k}{ }^{25}$. Measuring the coherent field spots in the quadrature $I-Q$ plane of the cavity field is projective for separated spots like those sketched in Fig. 1c, e. Hence, our proposed set-up enables to fuse pairs of MBSs. Strikingly, such a scheme also gives direct access to the fusion rules which are directly linked to the non-abelian algebra of the MBSs ${ }^{25}$. The full sequence for establishing the fusion rules is represented in Fig. 2 for an even total parity. One can measure the parity $\hat{P}_{12}$ and then $\hat{P}_{23}$ (panel a) or the parity $\hat{P}_{12}$ and then $\hat{P}_{34}$ (panel c). The results are expected to be qualitatively different whether $\hat{P}_{23}$ or $\hat{P}_{34}$ is measured. In the first case, random spots with equal weight should appear in the $I-Q$ plane along the axis defined by the first parity measurement whereas perfectly anti-correlated spots should appear in the second case. Specifically, the second parity measurement of the sequence of Fig. 2a shows that the fusion creates an equal weight superposition of states. Besides, the control sequence of Fig. $2 c$ can be used to establish that the system is not in a statistical mixture. It enables also to monitor the parity conservation. This should allow one to confirm that the state corresponding to two spots in the $I-Q$ plane is a coherent

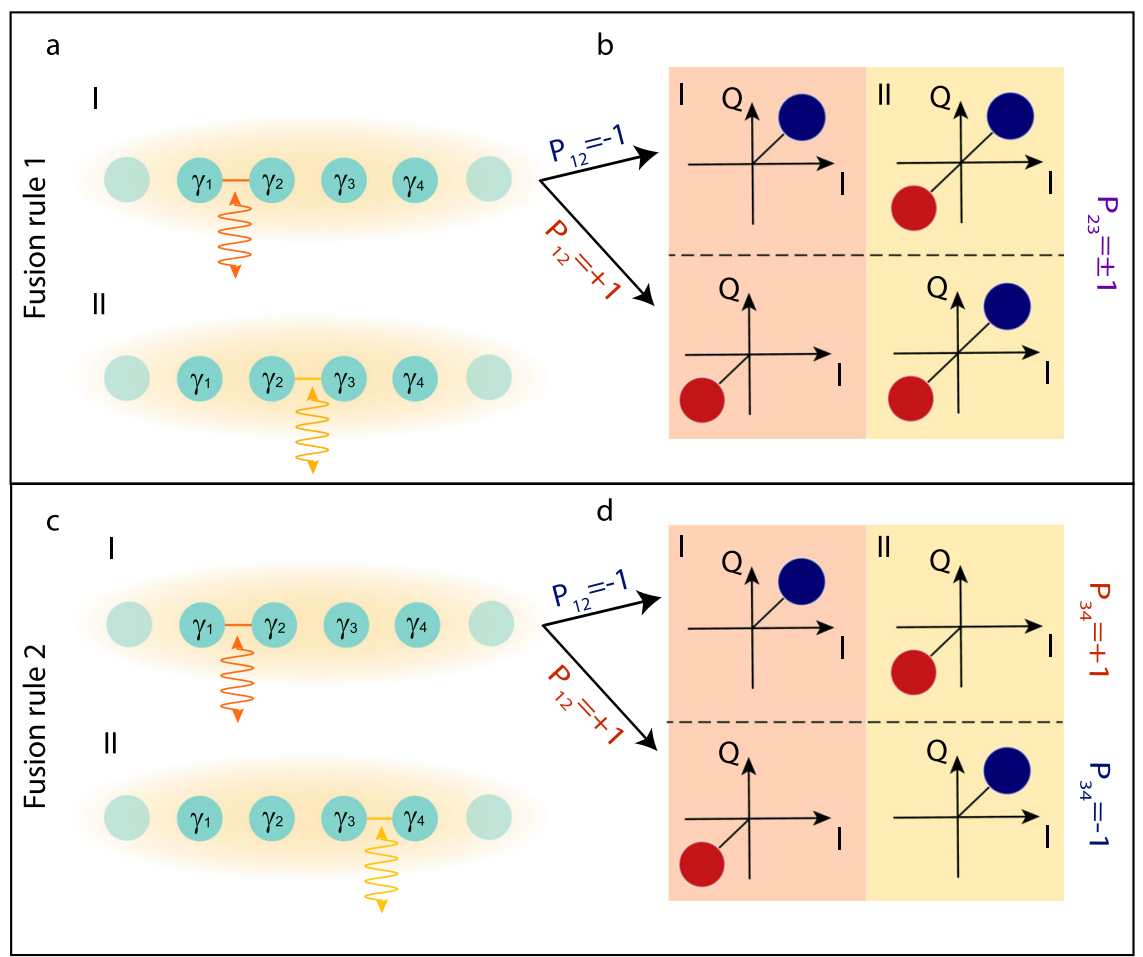

Fig. 2 Observing the fusion rules in the field quadrature plane. a Pulse sequences enabling the observation of the fusion rule of two adjacent Majorana modes. The successive pulses on the two adjacent sections of the physical chain can be tracked by coherent state spots in the quadrature $I-Q$ plane of the cavity field. $\mathbf{b}$ The fusion yields two spots of equal weight for both parities in the $I-Q$ plane starting from either of the two parities of section $(1,2)$. c Pulse sequence on the two separated sections of the physical chain. $\mathbf{d}$ The fusion yields anti-correlated spots for both parities in the $I-Q$ plane starting from either of the two parities. 
superposition. With this, we obtain a direct signature of the fusion rules of MBS1 and MBS2.

\section{Braiding protocol}

The braiding of two MBSs is the coherent exchange of them. Performing such an exchange in 1D is a challenge. It has been suggested to make use of anyon teleportation by strong parity measurements ${ }^{25-27}$ rather than moving in real space or in phase space the MBSs. However, this idea has not been implemented so far. One important roadblock is that one needs to read-out the parity corresponding to distant Majorana's such as 2 and 4 . The conventional wisdom is that this requires a network geometry since it seems difficult to "jump over" the intermediate Majorana (here Majorana 3) in a 1D set-up ${ }^{12}$. In our case, we can perform the braiding of 4 Majorana modes within one parity manifold of a six Majorana chain, using the additional two Majorana modes as a phase reference. The geometrical phase $\pm \pi / 4$ arising from clockwise/anticlockwise braiding has a measurable signature in the $I-Q$ plane of the cavity as shown in Fig. 3b. We start by the initialization of the state through the measurement of $\hat{P}_{46}$ and the postselection of the $\left|0_{46}\right\rangle$ parity state, starting from, e.g., $\left.\left|1_{12} 1_{34} 0_{56}\right\rangle\right)$. This gives the initial state $\left|\Psi_{\text {init }}\right\rangle=\frac{1}{\sqrt{2}}\left(\left|1_{12} 1_{34} 0_{56}\right\rangle+i\left|1_{12} 0_{34} 1_{56}\right\rangle\right)$, which we write in the natural basis formed by the eigenstates of $\hat{P}_{12}, \hat{P}_{34}$, and $\hat{P}_{56}$. The initial state $\left|\Psi_{\text {init }}\right\rangle$ creates a superposition of two different parities in the subspace associated with the four MBSs 1 to 4 . Since they live in different parity subspaces, they pick up opposite $\pi / 4$ phases during the braiding operation. The choice of the initial state and the pulse sequence makes them interfere like in a polarizer/analyzer set-up with birefringent media. The corresponding measurement sequences for $B_{14}$ and $B_{41}$ are displayed in Fig. 3a. For $B_{41}$, after the initialization sequence, one should measure $\hat{P}_{23}, \hat{P}_{21}$, then $\hat{P}_{24}$ and then $\hat{P}_{23}$, postselecting

a

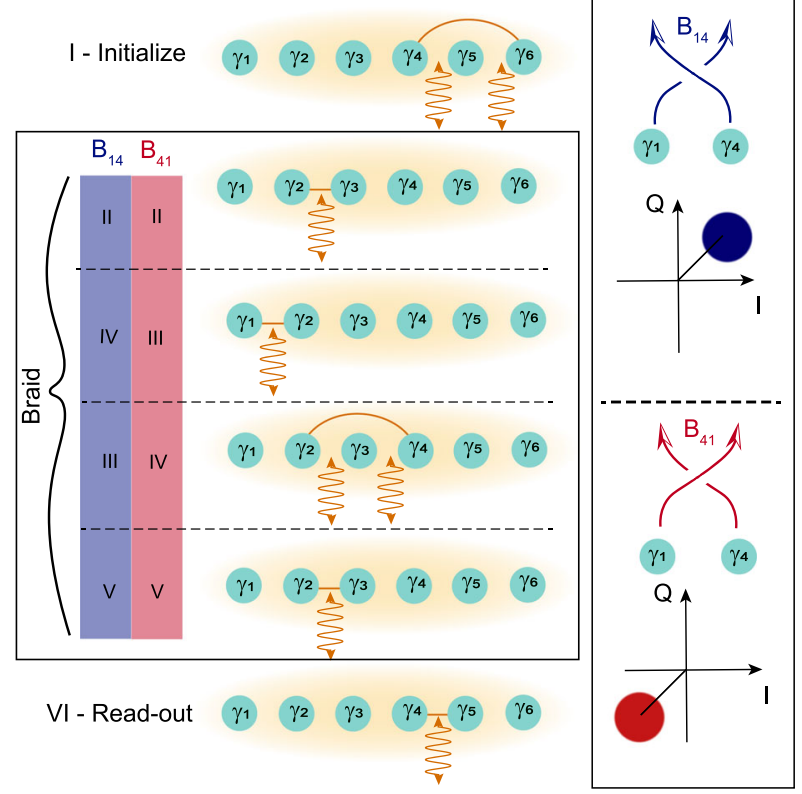

Fig. 3 Braiding protocol in a chain of six MBSs. a Pulse sequence enabling the "clockwise" or "counterclockwise" braiding depending on the order of pulse III or IV. The first pulse is an initialization and the last pulse is the readout. b Result of the clockwise and counterclockwise braiding as observed in the $I-Q$ plane trajectory of the coherent state spot (blue or red spots) after pulse VI. The qualitative difference of the cavity field in the two possible braids would be a direct observation of the non-abelian braiding. the +1 eigenvalue. For $B_{14}$, one should measure $\hat{P}_{23}, \hat{P}_{24}$, then $\hat{P}_{21}$ and then $\hat{P}_{23}$, postselecting the +1 eigenvalue. After the braiding (see "Methods"), we obtain the state $|\Psi\rangle_{\text {braided14 }}=$ $\frac{e^{-i \pi / 4}}{2}\left(\left|0_{12} 0_{34} 0_{56}\right\rangle+\left|1_{12} 1_{34} 0_{56}\right\rangle-\left|1_{12} 0_{34} 1_{56}\right\rangle-\left|0_{12} 1_{34} 1_{56}\right\rangle\right)$ for the clockwise braiding and $|\Psi\rangle_{\text {braided } 41}=\frac{e^{i \pi / 4}}{2}\left(\left|0_{12} 0_{34} 0_{56}\right\rangle+\right.$ $\left.\left|1_{12} 1_{34} 0_{56}\right\rangle+\left|1_{12} 0_{34} 1_{56}\right\rangle+\left|0_{12} 1_{34} 1_{56}\right\rangle\right)$ for the anti-clockwise braiding. The non-abelian character of the operation becomes therefore directly visible in the different outcomes of the coherent field spots in the $I-Q$ plane for the parity $\hat{P}_{45}$ measurement which is carried out at the last step in our protocol (see Fig. 3a). The clockwise braiding corresponds to the blue spot $\left(\hat{P}_{45}=-1\right)$ whereas the anti-clockwise braiding corresponds to the red spot $\left(\left(\hat{P}_{45}=+1\right)\right)$.

\section{T-gate}

The above methods for fusion or braiding can be extended to more complex gates. In particular, the T-gate (also called $\pi / 8$ gate) can be implemented in a 6 MBSs chain similar to that of Fig. 3. As the braiding of MBSs enables all the Clifford gates to be implemented, the T-gate presented here allows one to augment the Majorana platform for universal quantum computation ${ }^{28}$. It relies on a parity measurement involving simultaneously both the I and Q quadratures of the cavity field (i.e., along an arbitrary angle $\phi$ in the I-Q plane), each of them being coupled to $i \hat{\gamma}_{2} \hat{\gamma}_{3}$ and $i \hat{\gamma}_{3} \hat{\gamma}_{4}$ (more details can be found in the "Methods" section). Such a $\hat{P}_{\phi}$ measurement should be inserted in the place of the measurement of $\hat{P}_{24}$ in the sequence proposed for braiding. While such a gate is not topologically protected, it could be made exponentially accurate using mitigation techniques ${ }^{28}$.

\section{Stabilization of "imperfect" Majorana modes}

The previous discussion relies on the fact that we electrically manipulate, couple and read-out MBSs, which seems incompatible with topological protection because of electrical noise or disorder in the $\epsilon_{j j+1} \mathrm{~s}$. We now show another crucial consequence of the form (8) which implies that even for a chain of MBSs with finite overlap $\epsilon$ between the MBSs, one can induce with the cavity light a robust topological phase with stabilized, or self-corrected MBSs, i.e., with exponential protection. The principle of this exponential protection is to induce thanks to the cavity field and the gate modulation a synthetic, light-induced, Kitaev Hamiltonian as sketched in Fig. 4a. Like for error correction protocols ${ }^{29}$, this scheme requires some degree of redundancy and therefore longer chains than the ones considered so far. Let us first assume that we work with a chain with $N$ MBS sections $\{0 . . N\}$. We assume that a gate modulation is applied every other section, starting from section $(1,2)$. In such a condition, the Hamiltonian (8) becomes:

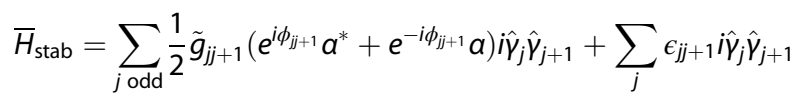

where $a$ is the classical part of the cavity field in the rotating frame and $\epsilon_{j j+1}$ is the residual overlap between physical MBSs. Assuming that the phases $\phi_{j j+1}$ and the modulations $\tilde{g}_{j j+1}$ are tuned to $\phi$ and $\tilde{g}$ and that the phase of the coherent field $a$ is $\theta, \bar{H}_{\text {stab }}$ can be divided into a Kitaev Hamiltonian $H_{\mathrm{K}}$ and a doping Hamiltonian $H_{\mathrm{D}}$ and has a topological phase transition with exponentially localized MBSs at sites 0 and $N$ (see Fig. 4a), for $J=\tilde{g}|a| \cos (\phi-\theta) \gg$ $-2 \epsilon_{j j+1}$ for all $j$ 's. These end MBSs are now stabilized because their overlap can be made exponentially small using macroscopic "knobs." The parameters $\tilde{g}$, $|a|$, and $\phi-\theta$ are these "knobs" and set the topological gap of our synthetic Kitaev Hamiltonian as shown in Fig. 4c. This principle can be used on bigger chains to produce 4-6 logical Majorana chains as needed by the previously introduced protocol. 
a

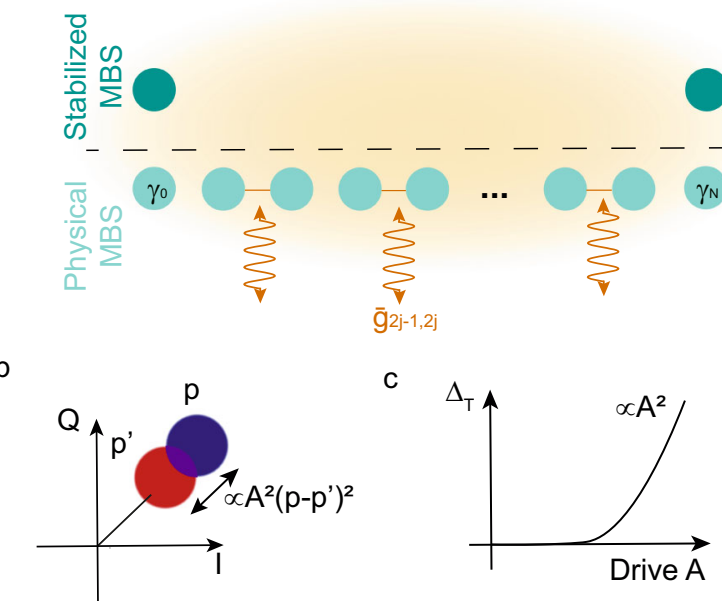

Fig. 4 Stabilized Majorana modes: topological and polaronic gaps. a Schematics of the Majorana chain in cavity with gate drives inducing the stabilization. The stabilized Majorana's are sketched in dark turquoise and stem from the resulting effective Kitaev chain. b Sketch of the polaronic protection with $A=\tilde{g} / 2\left(\omega_{\mathrm{RF}}-\omega_{\mathrm{c}}\right)$. c Topological gap as a function of the drive amplitude starting from a non-topological ground state.

\section{DISCUSSION}

In writing Hamiltonian (9), we have neglected two terms: one time-dependent classical field term $\delta H^{(1)}=\tilde{g}|a| \cos \left(2 \omega_{\mathrm{RF}} t+\phi+\right.$ $\theta) \sum_{j \text { odd }} i \hat{\gamma}_{j} \hat{\gamma}_{j+1}$ and one term arising from quantum fluctuations of the cavity field $\delta H^{(2)}=\tilde{g} \cos \left(\omega_{\mathrm{RF}} t+\phi-\theta\right) \sum_{j \text { odd }} i \hat{\gamma}_{j} \hat{\gamma}_{j+1}\left(\hat{b}+\hat{b}^{\dagger}\right)$. The quantum fluctuations of the cavity field are defined by the operator $\hat{b}$. Since both perturbations are periodic in time, it is convenient to use the Floquet formalism to study their effect (see "Methods" and Supplementary Note 1). Noting that all the parities $p_{j j+1}$ for the $(j, j+1)$ sections with $j$ odd are good quantum numbers in the Kitaev chain, the matrix elements arising in the perturbation theory depend now on $p=\Sigma p_{i j+1}$, which is an integer directly linked to the occupation of the chain and $m$ which is an integer arising from the Floquet ladder (see "Methods" and Supplementary Note 2). The first term is a fast oscillating term at roughly twice the cavity frequency. It generates matrix elements $\propto J_{\frac{m-m^{\prime}}{2}}\left(\frac{\left(p^{\prime}-p\right) \tilde{g}|a|}{2 \omega_{\mathrm{RF}}}\right)$ (see Supplementary Note 3). They can be safely neglected because they are of order $\left(\tilde{g}|a| / \omega_{\mathrm{RF}}\right)^{\frac{\left|m-m^{\prime}\right|}{2}}$ for small $\tilde{g}|a| / \omega_{\mathrm{RF}}$, which is a very realistic condition (see experimental requirements below). It is also essential to evaluate the effect of photonic quantum noise on the topological protection of our scheme. Defining the polaronic shift $E_{0}=\tilde{g}^{2} \omega_{\mathrm{c}} / 2\left(\omega_{\mathrm{RF}}^{2}-\omega_{\mathrm{c}}^{2}\right)$, we can write the quasi-energy of the driven chain as $: E_{\mathrm{K}}=J p+E_{0} p^{2}+$ $m \omega_{c}$. The result of perturbation theory on the Floquet space is twofold. First, any local perturbation $\eta$ flipping one of the $p_{j j+1}$ can only induce an exponentially small coupling between the end stabilized Majorana's at sites 0 and $N$ of order $\eta^{(N+1) / 2}$, thus preserving the topological protection. Second, the drive tends to shift the cavity field entangled with the state of the chain of quasienergy $E_{\mathrm{K}}$ at different spots in the $I-Q$ plane for different states of the chain with total quantum number $p$ or $p^{\prime}$ because $\delta H^{(2)}$ is a drive term proportional to $p$. The quasi-orthogonality of two coherent states with different amplitudes quenches exponentially the transition to excited states. The corresponding matrix element reads approximately: $\exp \left[-\frac{\tilde{g}^{2}}{8 \hbar^{2}\left(\omega_{\mathrm{RF}}-\omega_{\mathrm{c}}\right)^{2}}\left(p-p^{\prime}\right)^{2}\right]$ (Eq. (16) in the Supplementary). This exponential polaronic protection which further protects the topological phase is presented in Fig. $4 \mathrm{~b}$.

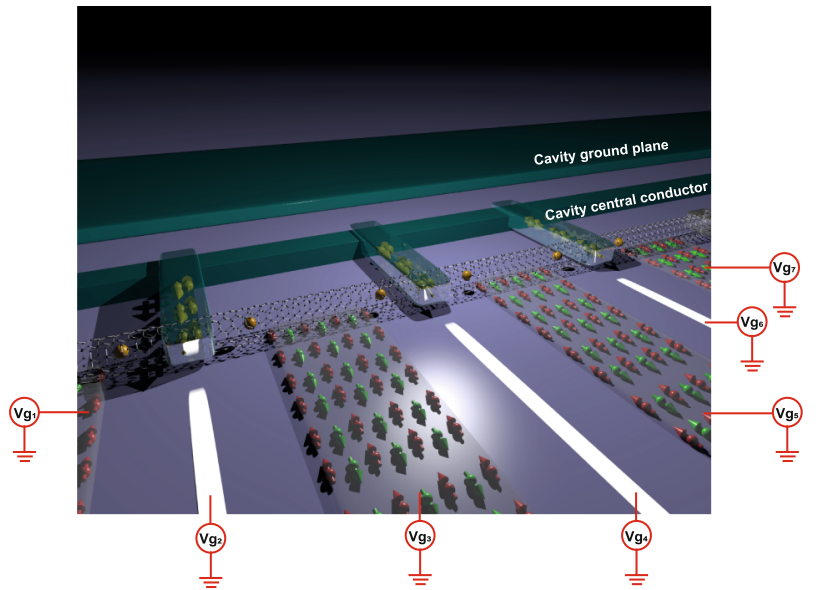

Fig. 5 Possible implementation. One possible physical implementation of our proposal for a six MBS chain. Here the topological phase is induced at zero magnetic field, i.e., easily compatible with microwave cavities by magnetic electrodes. The whole Majorana device is embedded in a superconducting coplanar waveguide cavity in green. The superconducting electrodes are galvanically coupled to the central conductor of the cavity. Gates 1, 3, 5, and 7 are magnetically textured. The white gates 2,4 , and 6 are nonmagnetic. The Majorana modes are the orange spheres.

We now consider here the experimental aspects related to our theoretical proposal. First of all, the nanoconductor may be implemented in various physical platforms. We give a possible implementation of the required set-up in Fig. 5. In this example, one uses a single wall carbon nanotube with several superconducting contacts and coupled to several magnetically textured gates providing a synthetic spin orbit interaction ${ }^{3}$. There are also several non-magnetic gates to tune the electrostatic potential profile in the carbon nanotube. The device is capacitively coupled to a superconducting high finesse microwave cavity. The use of a magnetic texture enables to drive the device in the topological phase without the use of an external magnetic field. Several set-ups have been implemented recently in this spirit ${ }^{3,30}$. This shows that the proposed set-up could be implemented without the need to apply a strong external magnetic field. In addition, it is worth mentioning that magnetic field resilient microwave cavities have been developed recently ${ }^{31}$, which enable the use of "conventional" Majorana set-ups, i.e., semiconducting nanowire-based set-ups in magnetic fields.

Since we use the tool box of cavity QED, the timescales for the gates, in particular the gate rise times should be in the nanosecond range ${ }^{16,17}$, which is within experimental reach. We also need to measure the cavity output in a time much smaller compared to the parity lifetime. The measurement time is of the order of a few $1 / \kappa$. Assuming $\kappa \approx 2 \pi \times 1 \mathrm{MHz}$ to be conservative and more than tens of microseconds for the parity lifetime, this means that the measurement can be performed in about $1 \mu \mathrm{s}$.

We then need to estimate how strongly the coupling strength can be modulated. In electrical circuits, coupling strength between a charge and a cavity of the order of $g=2 \pi \times$ $100 \mathrm{MHz}$ are now achievable ${ }^{32}$, and would still be compatible with the condition $\epsilon_{j j+1}, g_{j j+1} \ll \omega_{c}$. As shown in ref. ${ }^{19}$ where a specific semiconducting nanowire with overlapping MBSs was considered, such a magnitude of coupling strength can also lead to large Majorana-photon coupling for the physical Majorana's even if they are close to zero energy. Assuming that this coupling strength can by modulated by a factor $10 \%$ by using the electrostatic gates, our scheme enables single-shot cavity readout of the parity. 
Our set-up is generic in the sense that it works exactly the same way for the "non-stabilized" MBSs and the "stabilized" MBSs. The only difference is that the "stabilized" MBSs will have much smaller $g$ s than the physical ones far into the topological phase. For the parity read-out, which is at the basis of all the manipulations presented here, a small " $g$ " can be compensated by a small $K$ since the distance between the center of the $I-Q$ plane and the $+1 /-1$ parity spots scales like $g / \sqrt{\kappa}$ as shown in equation (22) of the input-output theory in the Methods section. Given the very small values of $\kappa \approx 2 \pi \times 1 \mathrm{kHz}$ which can be achieved in current technology for superconducting circuits, there is a lot of room to use our scheme for ultra-coherent MBSs. In the latter case, the use of an external magnetic field free platform ${ }^{3,30}$ will be more favorable to get a low $k$. For platforms hosting moderately coherent MBSs, a large " $g$ " might be more favorable and not too harmful for the coherence times, while enabling both stabilization and fast measurements in the $I-Q$ plane. While the complete timing of the measurement sequence will depend on the actual figures of merit of the experimental platform chosen to implement our scheme, we speculate that there is room for optimization.

In the stabilized version of the experiment, the value of the energy gap is of the order of $J$, which can be as large as $10 \mathrm{GHz}$, well above dilution refrigerator temperatures $20 \mathrm{mK}$ for $10^{6}$ photons in the cavity mode. In such a situation which is still within the boundaries for neglecting the off-resonant counterrotating terms of the RWA, it will be favorable to use a different cavity mode for the stabilization and the read-out/manipulation. Note that already for a Kitaev chain with a number of sites $\leq 10$, there are well-localized MBSs within a well-defined energy gap. This means that we expect a modest hardware overhead, essentially a $\times 5$, for implementing the full braiding protocol with 6 stabilized MBSs.

Finally, it is interesting to discuss the robustness to drive imperfections of the stabilization scheme. For example, although phase matching between all the section can be achieved technically by synchronizing several microwave generators, e.g., using the conventional atomic clock method, phase fluctuations can still arise. Under such conditions, the above system maps onto the disordered Kitaev chain. There are many studies devoted to that problem. It can be shown that provided the disorder is small compared to the hopping strength, the topological phase is preserved ${ }^{33}$. This corresponds to phase shifts smaller than $\pi$ in our case, which is definitely within experimental reach. A similar condition holds for inhomogeneities in the $g$ s along the chain.

In summary, we have presented CQED protocols based on the parametric modulation of light-matter coupling for performing advanced quantum gates for Majorana zero modes. Such an approach can also be used for a parametric stabilization of the Majorana zero modes, enhancing the topological protection of a given physical platform. This should allow one to perform advanced operations with exponentially protected Majorana zero modes.

\section{METHODS}

\section{Floquet formalism for the stabilized Majorana modes}

In deriving the effective Hamiltonian $\bar{H}_{\text {stab, }}$ the cavity field has been replaced by its resonant component in the rotating frame. As we have shown, this procedure generates a static Kitaev Hamiltonian $H_{\mathrm{K}}$. The goal of this section is to demonstrate that, crucially, the remarkable topological protection of Majorana edge mode degeneracy also extends to the periodically driven situation considered in the present work without relying on the rotating frame approximation.

We first write the cavity field as a sum:

$\hat{a}=|a| e^{-i\left(\omega_{\mathrm{RF}} t+\theta\right)}+\hat{b}$
Assuming that the coupling between the Majorana chain and the cavity is modulated only on the $(j, j+1)$ bonds with $j$ odd, we get the time-periodic coupling Hamiltonian:

$$
H_{\mathrm{c}}(t)=\sum_{j \text { odd }}\left(J+\tilde{J} \cos \left(2 \omega_{\mathrm{RF}} t+\phi+\theta\right)+\tilde{g} \cos \left(\omega_{\mathrm{RF}} t+\varphi\right)\left(\hat{b}+\hat{b}^{\dagger}\right)\right) i \hat{\gamma}_{j} \hat{\gamma}_{j+1}
$$

Here we have set $J=\tilde{J} \cos \varphi$, with $\tilde{J}=\tilde{g}|\alpha|$ and $\varphi=\phi-\theta$. This has the form:

$H_{\mathrm{c}}(t)=H_{\mathrm{K}}+\delta H^{(1)}(t)+\delta H^{(2)}(t)$.

Besides the static Kitaev Hamiltonian already derived earlier using the rotating wave approximation, we get two time-periodic perturbations $\delta H^{(1)}(t)$ and $\delta H^{(2)}(t)$. The former induces a time-periodic modulation of the Kitaev coupling, $J$ being replaced by $J_{\text {eff }}(t)=J+\tilde{J} \cos \left(2 \omega_{\mathrm{RF}} t+\phi+\theta\right)$. The later couples the Majorana modes to quantum fluctuations of the cavity field. A key feature of this model is that both $\delta H^{(1)}(t)$ and $\delta H^{(2)}(t)$ commute with $H_{\mathrm{K}}$, and even more importantly, with its local conserved operators $\hat{p}_{j j+1}=i \hat{\gamma}_{j} \hat{v}_{j+1}$ for odd $j$. Since the existence of conserved local operators lies at the heart of topological protection, the persistence of this property in the full $H_{c}(t)$ is of course essential for our purpose here.

The first key ingredient to achieve topological protection is a large energy gap, compared to the strength of the static perturbation $\epsilon_{j j+1}$. Here lies a potential fragility of the present proposal, because inelastic interactions due to the periodic driving may strongly reduce the value of the effective gap below its static value 2 J. This concern is particularly clear for the $\delta H^{(1)}(t)$ perturbation because $J_{\text {eff }}(t)$ vanishes twice in each period $\pi / \omega_{\mathrm{RF}}$ (or just once if $\varphi$ is an integer multiple of $\pi$ ).

To address this issue, we have to extend the analysis of topological protection to situations where the reference Hamiltonian is time-periodic. We should first understand the Floquet spectrum of $H_{c}(t)$ and then investigate the effect of the static perturbation $H_{\mathrm{D}}=\sum_{j} \epsilon_{j j+1} i \hat{\gamma}_{j} \hat{\gamma}_{j+1}$. To make the discussion clearer, we shall discuss separately the Floquet spectra when either $\delta H^{(1)}(t)$ or $\delta H^{(2)}(t)$ is added to $H_{\mathrm{k}}$.

Let us denote by $\left|\tau,\left\{p_{j j+1}\right\}\right\rangle$ a state of the Majorana chain such that:

$i \hat{\gamma}_{j} \hat{\gamma}_{j+1}\left|\tau,\left\{p_{j j+1}\right\}\right\rangle=p_{j j+1}\left|\tau,\left\{p_{j j+1}\right\}\right\rangle \quad(j$ odd $)$

$i \hat{\gamma}_{0} \hat{\gamma}_{N}\left|\tau,\left\{p_{j j+1}\right\}\right\rangle=\tau\left|\tau,\left\{p_{j j+1}\right\}\right\rangle$

Here each eigenvalue $p_{j j+1}$ and $\tau$ can be \pm 1 . The Floquet eigenstates of $H_{\mathrm{K}}+\delta H^{(1)}(t)$ have the form:

$|\Psi(t)\rangle=e^{-i p J t} e^{-i p j \frac{-\sin \left(2 \omega_{B} F++\phi+\theta\right)}{2 \omega_{R F}}}\left|\tau,\left\{p_{j j+1}\right\}\right\rangle$,

so their Floquet quasi-energy is $p J$, which is defined modulo $\hbar \omega_{\mathrm{RF}}$.

To study the effect of $H_{\mathrm{D}}$, we view it as a perturbation of the operator $\mathcal{L}_{1}=H_{\mathrm{K}}+\delta H^{(1)}(t)-i \frac{\mathrm{d}}{\mathrm{d} t^{\prime}}$ acting in the Hilbert space $\mathcal{H}_{\text {per }}$ of periodic wavefunctions of $t$ with period $T$. More details on this procedure are given in the Supplementary Note 1 section. From Eq. (15), a complete eigenvector basis for $\mathcal{L}_{1}$ is given by states $\left.\left|\tau,\left\{p_{j j+1}\right\} ; m\right\rangle\right\rangle$ with eigenvalues $p J-m \hbar \omega_{\mathrm{RF}}$.

Topological protection means that the effective coupling between Majorana end modes generated by the static perturbation $H_{\mathrm{D}}$ is exponentially small in $N$. The existence of the local conserved operators $\hat{p}_{i j+1}$ (for odd $j$ ) implies that such an effective coupling, proportional to $i \hat{Y}_{0} \hat{Y}_{N}$, occurs only at order $(N+1) / 2$ in perturbation theory. Indeed, the lowest-order product of Majorana operators that contains both $\hat{\gamma}_{0}$ and $\hat{\gamma}_{N}$, and that commutes with all $\hat{p}_{j j+1}$ operators (for odd $j$ ) is $\prod_{l} i \hat{\gamma}_{2 l} \hat{\gamma}_{2 l+1}$, where $I$ runs from 0 to $(N-1) / 2$. Each term of the product corresponds to a local perturbation $i \epsilon_{2 l, 2 l+1} \hat{\gamma}_{2 l} \hat{\gamma}_{2 l+1}$. Let us assume that it connects state $\left.\left|\tau,\left\{p_{j j+1}\right\} ; m\right\rangle\right\rangle$ to state $\left.\left|\tau^{\prime},\left\{p_{j j+1}^{\prime}\right\} ; m^{\prime}\right\rangle\right\rangle$. In this case, one has $p_{j j+1}^{\prime}= \pm p_{j j+1}$, the minus sign occurring only if $j=2 l-1$ or $j=2 l+1$. To each intermediate state is associated an energy denominator $\left(p_{\mathrm{GS}}-p\right) J+$ $m \hbar \omega_{\mathrm{RF}}$, where $p_{\mathrm{GS}}=-(N-1) / 2$ since $p_{j, j+1}=-1$ for any odd $j$ in any of the twofold degenerate ground states of $H_{k}$. Compared to the static case, we see that the large gap proportional to $J$ is replaced by the smaller value $\min _{\{m\}}\left(J-m \hbar \omega_{\mathrm{RF}}\right)$. Therefore, a necessary condition for topological protection to survive in the presence of a periodic modulation of $J_{\text {eff }}$ is that inelastic transitions to states with a non-zero value of $m$ should be strongly suppressed. It is thus crucial to examine in more detail the matrix elements of the perturbation. 
Using the time dependence of unperturbed Floquet eigenstates given by Eq. (15), we get:

$$
\left\langle\left\langle\tau^{\prime},\left\{p_{j j+1}^{\prime}\right\} ; m^{\prime}\left|i \hat{\gamma}_{2 l} \hat{Y}_{2 l+1}\right| \tau,\left\{p_{j j+1}\right\} ; m\right\rangle\right\rangle \propto J_{\frac{m-m^{\prime}}{2}}\left(\frac{\left(p^{\prime}-p\right) \tilde{\jmath}}{2 \omega_{\mathrm{RF}}}\right), \text { for } m^{\prime}-m \text { even, }
$$

and this matrix element vanishes if $m^{\prime}-m$ is odd. In Eq. (16), $J_{\frac{m-m^{\prime}}{2}}$ is the usual Bessel function of the first kind. Since the above matrix element is proportional to $\left(\tilde{J} / \omega_{\mathrm{RF}}\right)^{\frac{\left|m-m^{\prime}\right|}{2}}$ at small $\tilde{J} / \omega_{\mathrm{RF}}$, we see that inelastic transitions to states with a non-zero value of $m$ are suppressed when $\tilde{J}<<\omega_{\mathrm{RF}}$, i.e., when the driving frequency is large compared to the time averaged gap of the effective Kitaev chain.

Although this argument is quite compelling, a potential danger lies in the fact that the ordering between the $(N+1) / 2$ local perturbations $i \epsilon_{2 l, 2 l+1} \hat{\gamma}_{2 \mid} \hat{\gamma}_{2 l+1}$ is arbitrary, so we have $\left(\left(N^{2}-1\right) / 8\right)$ ! terms at order $(N+1) / 2$. In the static case, this factorial growth is compensated by the large value of typical energy denominators. In the periodically modulated case, no exact solution in the presence of the static perturbation $H_{\mathrm{D}}$ is available, and to establish rigorously that the effective coupling between boundary Majorana modes decays exponentially with $N$ would require a more involved analysis, which is beyond the scope of the present work.

Let us now turn to the Floquet eigenstates of $H_{K}+\delta H^{(2)}(t)$. Since this Hamiltonian commutes with the conserved operators of $H_{k}$, we can put the Majorana chain in one of the states $\left|\tau,\left\{p_{j j+1}\right\}\right\rangle$ for all times $t$. The quantum oscillator mode of the cavity is then described by the Hamiltonian:

$$
H_{\text {cav }}(t)=\hbar \omega_{\mathrm{c}} \hat{b}^{\dagger} \hat{b}+p \tilde{g} \cos \left(\omega_{\mathrm{RF}} t+\phi\right)\left(\hat{b}+\hat{b}^{\dagger}\right)
$$

The Floquet spectrum of $H_{\text {cav }}$ is discussed in the Supplementary Note 2 section. Let us first consider the non-resonant case, when the detuning $\delta=\omega_{\mathrm{c}}-\omega_{\mathrm{RF}}$ is larger than the cavity damping rate $\Gamma$. Combining the Majorana chain and the cavity, the eigenstates of the operator $\mathcal{L}_{2}=H_{\mathrm{K}}+\delta H^{(2)}(t)-i \frac{\mathrm{d}}{\mathrm{d} t^{\prime}}$ acting in the Hilbert space $\mathcal{H}_{\text {per }}$ can be written as $\left.\left|\tau,\left\{p_{i j+1}\right\} ; n ; m\right\rangle\right\rangle$, where $n$ is a non-negative integer associated with the cavity oscillator and, as before, $m$ labels Fourier modes in the auxiliary space of periodic functions of time. The corresponding eigenvalues are $p J+p^{2} E_{0}+n \hbar \omega_{\mathrm{c}}-m \hbar \omega_{\mathrm{RF}}$.

The effect of the static perturbation $H_{\mathrm{D}}$ is similar to the previous case. The local perturbation term $i \epsilon_{21,21+1} \hat{\gamma}_{2 l} \hat{\gamma}_{2 l+1}$ acts only on the Majorana chain, where it connects state $\left.\left|\tau,\left\{p_{i j+1}\right\} ; m\right\rangle\right\rangle$ to state $\left.\left|\tau^{\prime},\left\{p_{i j+1}^{\prime}\right\} ; m^{\prime}\right\rangle\right\rangle$. The new feature with $\delta H^{(2)}(t)$, compared to $\delta H^{(1)}(t)$, is that the transition between these two states of the chain also modifies the amplitude of the periodic driving seen by the cavity mode. The analysis of these matrix elements is presented in the Supplementary Note 3 in the case where the detuning $\delta$ is small. One of the main features is the approximate selection rule $n^{\prime}-m^{\prime}=n-m$. This implies that the energy denominators $\left(p_{\mathrm{GS}}-p\right) J+$ $\left(p_{\mathrm{GS}}^{2}-p^{2}\right) E_{0}-n \hbar \omega_{c}+m \hbar \omega_{\mathrm{RF}}$ in the leading contributions to the effective coupling between boundary Majorana modes are close to the values $\left(p_{\mathrm{GS}}-p\right) J$ governing the static case. This gives strong support to our claim that topological protection is achieved in this model of a driven Majorana chain. Another bonus provided by the driven model comes from the fact that the matrix elements of $H_{\mathrm{D}}$ are proportional to the overlap between coherent states:

$$
\left\langle z^{\prime}(t) \mid z(t)\right\rangle=\exp \left(-\frac{\left(p^{\prime}-p\right)^{2} \tilde{g}^{2}}{8 \hbar^{2} \delta^{2}}\right) .
$$

The Gaussian factor in Eq. (19) may be significantly $<1$, which would enhance the protection of the ground-state degeneracy with respect to residual static perturbations such as $H_{\mathrm{D}}$. This is analogous to the reduction of a polaron hopping amplitude, due to its strong coupling to lattice vibration modes. In this analogy, the polaron becomes the Majorana chain and the vibration modes are replaced by the cavity oscillator.

In the case of a finite cavity damping $\kappa$, it is necessary to take into account the coupling of the cavity oscillator to a continuum of environmental modes. In the limit of a small damping $\omega_{c} \gg k$, it is shown in the Supplementary Note 4 that the driving term in $H_{\text {cav }}(t)$ couples mostly to the dressed modes near the cavity frequency $\omega_{c}$. Therefore, most of the previous analysis of topological protection in the limit of small detuning survives in the case of a small but finite damping.

\section{Input-output theory}

We show here how one can capture the cavity-based measurement processes for the parity of the Majorana chain using an input-output theory. This method gives results that not only agree with Eq. (2) of the main text but it also allows to capture the dissipative dynamics related to the projective measurement of the system. The equations of motion for the photonic field in the cavity $\hat{a}$ with loss rate $\kappa$ and for the input and output fields, $\hat{a}_{\text {in }}$ and $\hat{a}_{\text {out }}$, read, for a pair of MBSs $(1,2)$ :

$$
\begin{aligned}
\frac{\mathrm{d} \hat{a}}{\mathrm{~d} t} & =\frac{-i}{\hbar}[\hat{a}, H]-\frac{\kappa}{2} \hat{a} \\
& =\frac{-i \tilde{g}_{L}}{2} \hat{P}_{12}-\frac{\kappa}{2} \hat{a}-\sqrt{\kappa} \hat{a}_{\text {in }} \\
\hat{a}_{\text {out }} & =\hat{a}_{\text {in }}+\sqrt{\kappa} \hat{a}
\end{aligned}
$$

with $\hat{P}_{12}(t)$ constant since $\left[H, \hat{P}_{12}\right]=0$.

In the semi-classical regime $(a=\langle\hat{a}\rangle)$ and in absence of a cavity drive, the output field is given by:

$a_{\text {out }}(t)=\frac{-i \tilde{g}_{12}}{\sqrt{\kappa}}<\hat{P}_{12}>\left(1-e^{-\kappa t / 2}\right)$.

The modulation of $g_{12}$ populates the cavity field, as represented in Fig. 1b. By measuring the occupation of the cavity along the appropriate quadrature (more specifically, with a measurement phase of $\left.\phi_{\text {meas }}=\phi_{12}+\pi / 2[\pi]\right)$, one can therefore perform a measurement of the parity. The signal-to-noise ratio (SNR) in this measurement depends on $\frac{g_{12}}{\sqrt{k}}$ and on the measurement time $\tau$. It is given by ${ }^{24}$ :

$\operatorname{SNR}=\frac{\sqrt{8}\left|\tilde{g}_{12}\right|}{\kappa} \sqrt{\kappa \tau}\left(1-\frac{2}{\kappa \tau}\left(1-e^{-\kappa \tau / 2}\right)\right)$

We now show how the parity $\hat{P}_{24}$ can be measured as a dispersive shift of the cavity resonant frequency as explained in the main text, using:

$g_{23(34)}(t)=\bar{g}_{23(34)}+\tilde{g}_{23(34)} \cos \left(\omega_{R F} t+\phi_{23(34)}\right)$

The coupled equations of motion are:

$\frac{\mathrm{d} \hat{a}}{\mathrm{~d} t}=-i \omega_{\mathrm{c}} \hat{a}-i g_{23}(t) \hat{\gamma}_{2} \hat{\gamma}_{3}-i g_{34}(t) \hat{\gamma}_{3} \hat{\gamma}_{4}-\kappa / 2$

$\frac{\mathrm{d} \hat{\gamma}_{2} \hat{\gamma}_{3}}{\mathrm{~d} t}=-2 i g_{34}(t) \hat{\gamma}_{2} \hat{\gamma}_{4}\left(\hat{a}+\hat{a}^{\dagger}\right)$

$\frac{\mathrm{d} \hat{\gamma}_{3} \hat{\gamma}_{4}}{\mathrm{~d} t}=2 i g_{23}(t) \hat{\gamma}_{2} \hat{\gamma}_{4}\left(\hat{a}+\hat{a}^{\dagger}\right)$

In the rotating frame $\left(a t \omega_{c}\right)$, and keeping resonant terms in the RWA, we get a first reduced equation on the cavity field:

$\frac{\mathrm{d} \hat{a}}{\mathrm{~d} t}=-\frac{\kappa}{2} \hat{a}-2 \int \mathrm{d} \tau \hat{\gamma}_{2}(\tau) \hat{\gamma}_{4}(\tau)\left(g_{23}(t) g_{34}(\tau)-g_{34}(t) g_{23}(\tau)\right)\left(\hat{a}+\hat{a}^{\dagger}\right)$

We additionally suppose $\left|\omega_{\mathrm{RF}}-\omega_{\mathrm{c}}\right|<<\omega_{\mathrm{c}}$ so that we can neglect the time evolution of $\hat{\gamma}_{2} \hat{\gamma}_{4}$, as well as the one of $\hat{a}$ in the above integral. This gives:

$\frac{\mathrm{d} \hat{a}}{\mathrm{~d} t}=-4 \hat{\gamma}_{2} \hat{\gamma}_{4} \hat{a} \frac{\tilde{g}_{23} \tilde{g}_{34}}{\omega_{\mathrm{c}}-\omega_{\mathrm{RF}}} \sin \left(\phi_{34}-\phi_{23}\right)-\frac{\kappa}{2} \hat{a}$

One sees here again that the optimum is $\phi_{34}-\phi_{23}=\pi / 2$.

\section{Distinction with accidental Andreev bound states}

It is important to stress that our set-up can also distinguish between Majorana modes and accidental Andreev bound states. Whereas braiding is a priori the most unambiguous way of distinguishing between accidental Andreev bound states and Majorana modes, establishing the fusion rules should be enough for a large class of situations. An Andreev bound state is expected to give rise to a transverse coupling that yields a trajectory of the type of Fig. 1e. Measuring several sections of the chain which display only trajectories of the type of Fig. $1 \mathrm{c}$ in the $I-Q$ plane (i.e., longitudinal coupling) in the fusion rule set-up (with four nodes) should constraint very much the models with accidental Andreev bound states (if any exists) yielding the same signature. 


\section{State sequence in the braiding protocol}

We recall first the measurement-based braiding protocol and specifically apply it to our scheme. Let us consider again a linear chain of four Majorana quasiparticles, $\hat{\gamma}_{j=1.4}$. The set of measurements needed for performing a braiding operation $\hat{B}_{14}$ between two Majorana $\hat{\gamma}_{1}$ and $\hat{\gamma}_{4}$ stems from the identity ${ }^{12,26}$ :

$\hat{\Pi}_{23} \hat{\Pi}_{21} \hat{\Pi}_{24} \hat{\Pi}_{23}=\frac{1}{\sqrt{8}} \hat{\Pi}_{23} \hat{B}_{14}$

$\hat{\Pi}_{j k}=\frac{1}{2}\left(1+\hat{P}_{j k}\right)$

The operator $\hat{\Pi}_{j k}$ projects the electronic state onto the subspace with parity $\hat{P}_{j k}=1$.

For the state sequence presented in this work, we start by measuring $\hat{P}_{46}$ giving an initial state $\left|\Psi_{\text {init }}\right\rangle=\frac{1}{\sqrt{2}}\left(\left|1_{12} 1_{34} 0_{56}\right\rangle+i\left|1_{12} 0_{34} 1_{56}\right\rangle\right)$, postselecting the $\left|0_{46}\right\rangle$ parity state, starting from $\left|1_{12} 1_{34} 0_{56}\right\rangle$.

Then, for the state sequence of $\hat{B}_{41}$, we project onto the +1 eigenvalue using the projection operators $\hat{\Pi}_{23}, \hat{\Pi}_{21}, \hat{\Pi}_{24}$, and then $\hat{\Pi}_{23}$. This gives the sequence of states:

$\left|\Psi_{1}\right\rangle=\hat{\Pi}_{23}\left|\Psi_{\text {init }}\right\rangle=\frac{1}{2}\left(\left|0_{12} 0_{34} 0_{56}\right\rangle+\left|1_{12} 1_{34} 0_{56}\right\rangle+i\left|1_{12} 0_{34} 1_{56}\right\rangle+i\left|0_{12} 1_{34} 1_{56}\right\rangle\right)$

$\left|\Psi_{11}\right\rangle=\hat{\Pi}_{21}\left|\Psi_{1}\right\rangle=\frac{1}{\sqrt{2}}\left(\left|1_{12} 1_{34} 0_{56}\right\rangle+i\left|1_{12} 0_{34} 1_{56}\right\rangle\right)$

$\left|\Psi_{\text {III }}\right\rangle=\hat{\Pi}_{24}\left|\Psi_{11}\right\rangle=\frac{1}{2}\left(i\left|0_{12} 0_{34} 0_{56}\right\rangle+\left|1_{12} 1_{34} 0_{56}\right\rangle+i\left|1_{12} 0_{34} 1_{56}\right\rangle+\left|0_{12} 1_{34} 1_{56}\right\rangle\right)$

$|\Psi\rangle_{\text {braided } 41}=\left|\Psi_{\text {IV }}\right\rangle=\hat{\Pi}_{23}\left|\Psi_{\text {III }}\right\rangle=\frac{e^{i \pi / 4}}{2}\left(\left|0_{12} 0_{34} 0_{56}\right\rangle+\left|1_{12} 1_{34} 0_{56}\right\rangle\right.$

$$
\left.+\left|1_{12} 0_{34} 1_{56}\right\rangle+\left|0_{12} 1_{34} 1_{56}\right\rangle\right)
$$

The state sequence for $\hat{B}_{14}$ is:

$\left|\Psi_{1}\right\rangle=\hat{\Pi}_{23}\left|\Psi_{\text {init }}\right\rangle=\frac{1}{2}\left(\left|0_{12} 0_{34} 0_{56}\right\rangle+\left|1_{12} 1_{34} 0_{56}\right\rangle+i\left|1_{12} 0_{34} 1_{56}\right\rangle+i\left|0_{12} 1_{34} 1_{56}\right\rangle\right)$

$\left|\Psi_{11}\right\rangle=\hat{\Pi}_{24}\left|\Psi_{1}\right\rangle=\frac{e^{i \pi / 4}}{2}\left(\left|0_{12} 0_{34} 0_{56}\right\rangle-i\left|1_{12} 1_{34} 0_{56}\right\rangle+i\left|1_{12} 0_{34} 1_{56}\right\rangle+\left|0_{12} 1_{34} 1_{56}\right\rangle\right)$

$\left|\Psi_{11}\right\rangle=\hat{\Pi}_{12}\left|\Psi_{11}\right\rangle=\frac{e^{-i \pi / 4}}{2}\left(\left|1_{12} 1_{34} 0_{56}\right\rangle-\left|1_{12} 0_{34} 1_{56}\right\rangle\right)$

$$
\begin{aligned}
|\Psi\rangle_{\text {braided 14 }}=\left|\Psi_{\text {IV }}\right\rangle=\hat{\Pi}_{23}\left|\Psi_{\text {III }}\right\rangle= & \frac{e^{-i \pi / 4}}{2}\left(\left|0_{12} 0_{34} 0_{56}\right\rangle+\left|1_{12} 1_{34} 0_{56}\right\rangle\right. \\
& \left.-\left|1_{12} 0_{34} 1_{56}\right\rangle-\left|0_{12} 1_{34} 1_{56}\right\rangle\right)
\end{aligned}
$$

We therefore arrive at the result of the main text: $|\Psi\rangle_{\text {braided } 14}$ is an eigenvector of $\hat{P}_{45}$ for the clockwise braiding with eigenvalue -1 , yielding the blue spot in the $I-Q$ plane and $|\Psi\rangle_{\text {braided } 41}$ is an eigenvector of $\hat{P}_{45}$ for the anti-clockwise braiding with eigenvalue +1 , yielding the red spot in the $I-Q$ plane. The reasoning for the even total parity is exactly the same.

\section{DATA AVAILABILITY}

The full details of the calculations present in the presented work are available from the corresponding authors upon reasonable request.

Received: 5 November 2020; Accepted: 13 November 2021; Published online: 16 December 2021

\section{REFERENCES}

1. Albrecht, S. et al. Exponential protection of zero modes in Majorana islands. Nature 531, 206-209 (2016).

2. Gül, O. et al. Ballistic Majorana nanowire devices. Nat. Nanotechnol. 13, 192-197 (2018).
3. Desjardins, M. M. et al. Synthetic spin orbit interaction for Majorana devices. Nat. Mater. 18, 1060-1064 (2019).

4. Ren, H. et al. Topological superconductivity in a phase-controlled Josephson junction. Nature 569, 93-98 (2019).

5. Fornieri, A. et al. Evidence of topological superconductivity in planar Josephson junctions. Nature 569, 89-92 (2019).

6. Alicea, J., Oreg, Y., Refael, G., von Oppen, F. \& Fisher, M. P. A. Non-abelian statistics and topological quantum information processing in 1D wire networks. Nat. Phys. 7, 412-417 (2011).

7. Hassler, F., Akhmerov, A. R. \& Beenakker, C. W. J. The top-transmon: a hybrid superconducting qubit for parity-protected quantum computation. N. J. Phys. 13, 095004 (2011).

8. van Heck, B., Akhmerov, A. R., Hassler, F., Burrello, M. \& Beenakker, C. W. J. Coulomb-assisted braiding of Majorana fermions in a Josephson junction array. N. J. Phys. 14, 035019 (2012).

9. Hyart, T. et al. Flux-controlled quantum computation with Majorana fermions. Phys. Rev. B 88, 035121 (2013).

10. You, J. Q., Wang, Z. D., Zhang, W. \& Nori, F. Encoding a qubit with Majorana modes in superconducting circuits. Sci. Rep. 4, 5535 (2014).

11. Aasen, D. et al. Milestones toward Majorana-based quantum computing. Phys. Rev. X 6, 031016 (2016).

12. Vijay, S. \& Fu, L. Teleportation-based quantum information processing with Majorana zero modes. Phys. Rev. B 94, 235446 (2016).

13. Karzig, T. et al. Scalable designs for quasiparticle-poisoning-protected topological quantum computation with Majorana zero modes. Phys. Rev. B 95, 235305 (2017).

14. Knapp, C., Väyrynen, J. I. \& Lutchyn, R. M. Number conserving analysis of measurement-based braiding with Majorana zero modes. Phys. Rev. B 101, 125108 (2020)

15. Clerk, A. A., Devoret, M. H., Girvin, S. M., Marquardt, F. \& Schoelkopf, R. J. Introduction to quantum noise, measurement, and amplification. Rev. Mod. Phys. 82, 1155-1208 (2010)

16. Hays, M. et al. Continuous monitoring of a trapped superconducting spin. Nat. Phys. 16, 1103-1107 (2020).

17. Hays, M. et al. Coherent manipulation of an Andreev spin qubit. Science 373 430-433 (2021).

18. Dartiailh, M. C., Kontos, T., Douçot, B. \& Cottet, A. Direct cavity detection of Majorana pairs. Phys. Rev. Lett. 118, 126803 (2017).

19. Cottet, A., Kontos, T. \& Douçot, B. Squeezing light with Majorana fermions. Phys. Rev. B 88, 195415 (2013).

20. Dmytruk, O., Trif, M. \& Simon, P. Cavity quantum electrodynamics with mesoscopic topological superconductors. Phys. Rev. B 92, 245432 (2015).

21. Müller, C., Bourassa, J. \& Blais, A. Detection and manipulation of Majorana fermions in circuit QED. Phys. Rev. B 88, 235401 (2013).

22. Trif, M. \& Simon, P. Braiding of Majorana fermions in a cavity. Phys. Rev. Lett. 122, 236803 (2019).

23. Cottet, A. et al. Cavity QED with hybrid nanocircuits: from atomic-like physics to condensed matter phenomena. J. Phys. Condens. Matter 29, 433002 (2017).

24. Didier, N., Bourassa, J. \& Blais, A. Fast quantum nondemolition readout by parametric modulation of longitudinal qubit-oscillator interaction. Phys. Rev. Lett. 115, 203601 (2015).

25. Beenakker, C. W. J. Search for non-Abelian Majorana braiding statistics in superconductors. Lecture Notes of Les Houches summer school on Quantum Machines (2019).

26. Bonderson, P., Freedman, M. \& Nayak, C. Measurement-only topological quantum computation. Phys. Rev. Lett. 101, 010501 (2008).

27. Zilberberg, O., Braunecker, B. \& Loss, D. Controlled-NOT for multiparticle qubits and topological quantum computation based on parity measurements. Phys. Rev. A 77, 012327 (2008).

28. Karzig, T., Oreg, Y., Refael, G. \& Freedman, M. H. Robust Majorana magic gates via measurements. Phys. Rev. B 99, 144521 (2019).

29. Litinski, D., Kesselring, M. S., Eisert, J. \& von Oppen, F. Combining topological hardware and topological software: color-code quantumcomputing with topological superconductor networks. Phys. Rev. X 7, 031048 (2019).

30. Vaitiekenas, S., Liu, Y., Krogstrup, P. \& Marcus, C. Zero-field topological superconductivity in ferromagnetic hybrid nanowires. Nat. Phys. 17, 43-47 (2021).

31. Kroll, J. G., Borsoi, F. \& van der Enden, K. L. Magnetic field resilient superconducting coplanar waveguide resonators for hybrid CQED experiments. Phys. Rev. Appl. 11, 064053 (2019).

32. Stockklauser, A. et al. Strong coupling cavity QED with gate-defined double quantum dots enabled by a high impedance resonator. Phys. Rev. X 7, 011030 (2017).

33. McGinley, M., Knolle, J. \& Nunnenkamp, A. Robustness of Majorana edge modes and topological order - exact results for the symmetric interacting Kitaev chain with disorder. Phys. Rev. B 96, 241113 (2017). 


\section{ACKNOWLEDGEMENTS}

We would like to thank J. Klinovaja, F. von Oppen, and N. Regnault for fruitful discussions. This work was supported by the Quantera project SuperTop.

\section{AUTHOR CONTRIBUTIONS}

L.C.C., M.R.D., A.C., and T.K. devised theoretically the parity measurement, the fusion, and the braiding protocol. B.D. developed the Floquet theory for the chain of Majorana's and the stabilization. All the authors co-wrote the manuscript.

\section{COMPETING INTERESTS}

Some of the authors (L.C.C., M.R.D., A.C., and T.K.) have filed a provisional patent for the braiding protocol described in the paper (patent application number 2009318).

\section{ADDITIONAL INFORMATION}

Supplementary information The online version contains supplementary material available at https://doi.org/10.1038/s41534-021-00508-w.

Correspondence and requests for materials should be addressed to A. Cottet or T. Kontos.
Reprints and permission information is available at http://www.nature.com/ reprints

Publisher's note Springer Nature remains neutral with regard to jurisdictional claims in published maps and institutional affiliations.

(i) Open Access This article is licensed under a Creative Commons Attribution 4.0 International License, which permits use, sharing, adaptation, distribution and reproduction in any medium or format, as long as you give appropriate credit to the original author(s) and the source, provide a link to the Creative Commons license, and indicate if changes were made. The images or other third party material in this article are included in the article's Creative Commons license, unless indicated otherwise in a credit line to the material. If material is not included in the article's Creative Commons license and your intended use is not permitted by statutory regulation or exceeds the permitted use, you will need to obtain permission directly from the copyright holder. To view a copy of this license, visit http://creativecommons. org/licenses/by/4.0/.

(c) The Author(s) 2021 